\title{
No evidence of amplified Plasmodium falciparum plasmepsin II gene copy number in an area with artemisinin-resistant malaria along the China-Myanmar border
}

\author{
Fang Huang ${ }^{1^{*} \mathbb{D}}$, Biraj Shrestha ${ }^{3}$, Hui Liu ${ }^{2}$, Lin-Hua Tang ${ }^{1}$, Shui-Sen Zhou' ${ }^{1}$ Xiao-Nong Zhou'
} Shannon Takala-Harrison ${ }^{3}$, Pascal Ringwald ${ }^{4}$, Myaing M. Nyunt ${ }^{5}$ and Christopher V. Plowe ${ }^{5}$

\begin{abstract}
Background: The emergence and spread of artemisinin resistance in Plasmodium falciparum poses a threat to malaria eradication, including China's plan to eliminate malaria by 2020. Piperaquine (PPQ) resistance has emerged in Cambodia, compromising an important partner drug that is widely used in China in the form of dihydroartemisinin (DHA)-PPQ. Several mutations in a P. falciparum gene encoding a kelch protein on chromosome 13 (k13) are associated with artemisinin resistance and have arisen spread in the Great Mekong subregion, including the China-Myanmar border. Multiple copies of the plasmepsin I/III (pm2/3) genes, located on chromosome 14, have been shown to be associated with PPQ resistance.

Methods: The therapeutic efficacy of DHA-PPQ for the treatment of uncomplicated $P$. falciparum was evaluated along the China-Myanmar border from 2010 to 2014. The dry blood spots samples collected in the efficacy study prior DHA-PPQ treatment and from the local hospital by passive detection were used to amplify $k 13$ and pm2. Polymorphisms within $k 13$ were genotyped by capillary sequencing and pm2 copy number was quantified by relativequantitative real-time polymerase chain reaction. Treatment outcome was evaluated with the World Health Organization protocol. A linear regression model was used to estimate the association between the day 3 positive rate and $k 13$ mutation and the relationship of the pm2 copy number variants and $k 13$ mutations.
\end{abstract}

Results: DHA-PPQ was effective for uncomplicated P. falciparum infection in Yunnan Province with cure rates $>95 \%$. Twelve non synonymous mutations in the $k 13$ domain were observed among the 268 samples with the prevalence of $44.0 \%$ and the predominant mutation was $\mathrm{F} 446 \mathrm{l}$ with a prevalence of $32.8 \%$. Only one sample was observed with multi-copies of $p m 2$, including parasites with and without $k 13$ mutations. The therapeutic efficacy of DHA-PPQ was > 95\% along the China-Myanmar border, consistent with the lack of amplification of pm2.

Conclusion: DHA-PPQ for uncomplicated P. falciparum infection still showed efficacy in an area with artemisininresistant malaria along the China-Myanmar border. There was no evidence to show PPQ resistance by clinical study and molecular markers survey. Continued monitoring of the parasite population using molecular markers will be important to track emergence and spread of resistance in this region.

\footnotetext{
*Correspondence: huangfang@nipd.chinacdc.cn

${ }^{1}$ National Institute of Parasitic Diseases, Chinese Center for Disease

Control and Prevention, Shanghai, People's Republic of China

Full list of author information is available at the end of the article
}

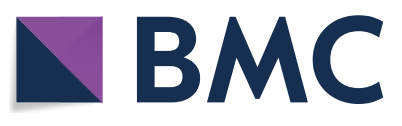

(c) The Author(s) 2020. This article is licensed under a Creative Commons Attribution 4.0 International License, which permits use, sharing, adaptation, distribution and reproduction in any medium or format, as long as you give appropriate credit to the original author(s) and the source, provide a link to the Creative Commons licence, and indicate if changes were made. The images or other third party material in this article are included in the article's Creative Commons licence, unless indicated otherwise in a credit line to the material. If material is not included in the article's Creative Commons licence and your intended use is not permitted by statutory regulation or exceeds the permitted use, you will need to obtain permission directly from the copyright holder. To view a copy of this licence, visit http://creativeco mmons.org/licenses/by/4.0/. The Creative Commons Public Domain Dedication waiver (http://creativecommons.org/publicdomain/ zero/1.0/) applies to the data made available in this article, unless otherwise stated in a credit line to the data. 
Keywords: Plasmodium falciparum, Artemisinin resistance, Piperaquine, Plasmepsin II, China-Myanmar border

\section{Background}

Malaria remains one of the global major public health problems. According to the latest world malaria report, there were an estimated 228 million cases and 405,000 deaths from malaria globally in 2018, compared with 416,000 estimated deaths in 2017 and 585,000 in 2010 [1].

Artemisinin-based combination therapy (ACT), which combines a fast-acting, rapidly eliminated artemisinin derivative with another slower-acting partner drug with a longer half-life, have played an indispensable role in reducing global malaria-associated mortality and morbidity [2]. Currently, five artemisinin-based combinations are recommended by the World Health Organization (WHO): artesunate-amodiaquine, artemether-lumefantrine, artesunate-mefloquine, artesunate-sulfadoxinepyrimethamine, and dihydroartemisinin-piperaquine (DHA-PPQ) [3]. ACT has been integral to the recent success of global malaria control and protecting its efficacy for the treatment of malaria has been a global health priority. However, the emergence and spread of artemisinin resistance in Plasmodium falciparum poses a threat to malaria control and eradication goals in the Greater Mekong subregion (GMS), where resistance has emerged independently and spread [4-7]. The WHO has implemented a strategy to eliminate $P$. falciparum from the six countries located in the GMS by 2025 to respond the threat of an untreatable multi-drug resistant parasite [8].

Several mutations in a $P$. falciparum gene encoding a kelch protein on chromosome $13(k 13)$ are associated with artemisinin resistance and have arisen multiple times and spread in the GMS, including the China-Myanmar border [9]. Over 200 nonsynonymous k13 mutations have been reported to date, of which nine variants (F446I, N458Y, M476I, Y493H, R539T, I543T, P553L, R561H, and C580Y) have been validated, and over $20 \mathrm{k} 13$ mutations are considered candidates or associated markers [9].

From 2010, PPQ became a partner drug with DHA to treat falciparum malaria recommended by the WHO [10]. DHA-PPQ, as the first-line drug for uncomplicated P. falciparum treatment, is widely used in China [11] and high efficacy has been reported [12-15]. Recently, the emergence of DHA-PPQ resistance was observed in Cambodia with high treatment failure rate and recrudescent infections. Resistance then rapidly spread to other countries in Southeast Asia [16-22], and subsequent investigations confirmed the presence of high treatment failure and PPQ resistance in Cambodia and Vietnam $[16,21,23]$. The evidence prompted a treatment policy change in Cambodia and Vietnam from DHA-PPQ to other artemisinin-based combinations in areas where DHA-PPQ was failing. Multiple copies of the P. falciparum plasmepsin II (pm2) genes (PF3D7_1408000), located on chromosome 14, encoding a protease involved in haemoglobin degradation, have recently been shown to be associated with PPQ resistance in Cambodia [19, 24]. This situation raises concern about resistance to the partner drugs of ACT.

There had been zero indigenous malaria infections in China from 2017 and China has vowed to interrupt local malaria transmission by 2020 [25]. Yunnan Province located in Southern China, bordering Myanmar, Laos and Vietnam is the key focus of the national malaria elimination program. In this study, we investigated the therapeutic efficacy of DHA-PPQ for treatment of uncomplicated $P$. falciparum along the China-Myanmar borders from 2010 to 2014. Sequencing of the propeller domain of the $k 13$ gene was performed to identify mutations and $p m 2$ copy number was quantified to determine the copy variants from both parasites with and without $k 13$ mutations.

\section{Methods \\ Study sites and design}

The clinical studies were one-arm prospective evaluations to observe treatment of uncomplicated malaria using a standard WHO therapeutic efficacy study (TES) protocol. The studies were conducted in four counties (Yingjiang, Tengchong, Menglian and Ruili) in Southwest Yunnan along the China-Myanmar border between 2010 and 2014 (Fig. 1).

\section{Recruitment of patients and follow up}

Patients aged $>6$ months with fever (axillary temperature $\geq 37.5{ }^{\circ} \mathrm{C}$ ) or a history of fever in the previous $48 \mathrm{~h}$ were screened for inclusion. Inclusion criteria were: mono-infection with $P$. falciparum; parasitaemia density between 500 and 100,000 asexual parasites/ $\mu$ l; no history of anti-malarial use in the past 14 days and no signs of severe malaria or danger signs. After written informed consent was provided, a detailed medical history, clinical examination and blood smears were performed for each participant. DHA-PPQ (Zhejiang Holley Nanhu Pharmaceutical Co. Ltd, China) was administered at a total adult dose of $2.5 \mathrm{mg} / \mathrm{kg}$ dihydroartemisnin and $20 \mathrm{mg} / \mathrm{kg} \mathrm{PPQ}$ for 3 days. All the anti-malarial drugs were provided by the WHO.

After the first day of treatment (day 0), clinical and laboratory tests, including axillary temperature 


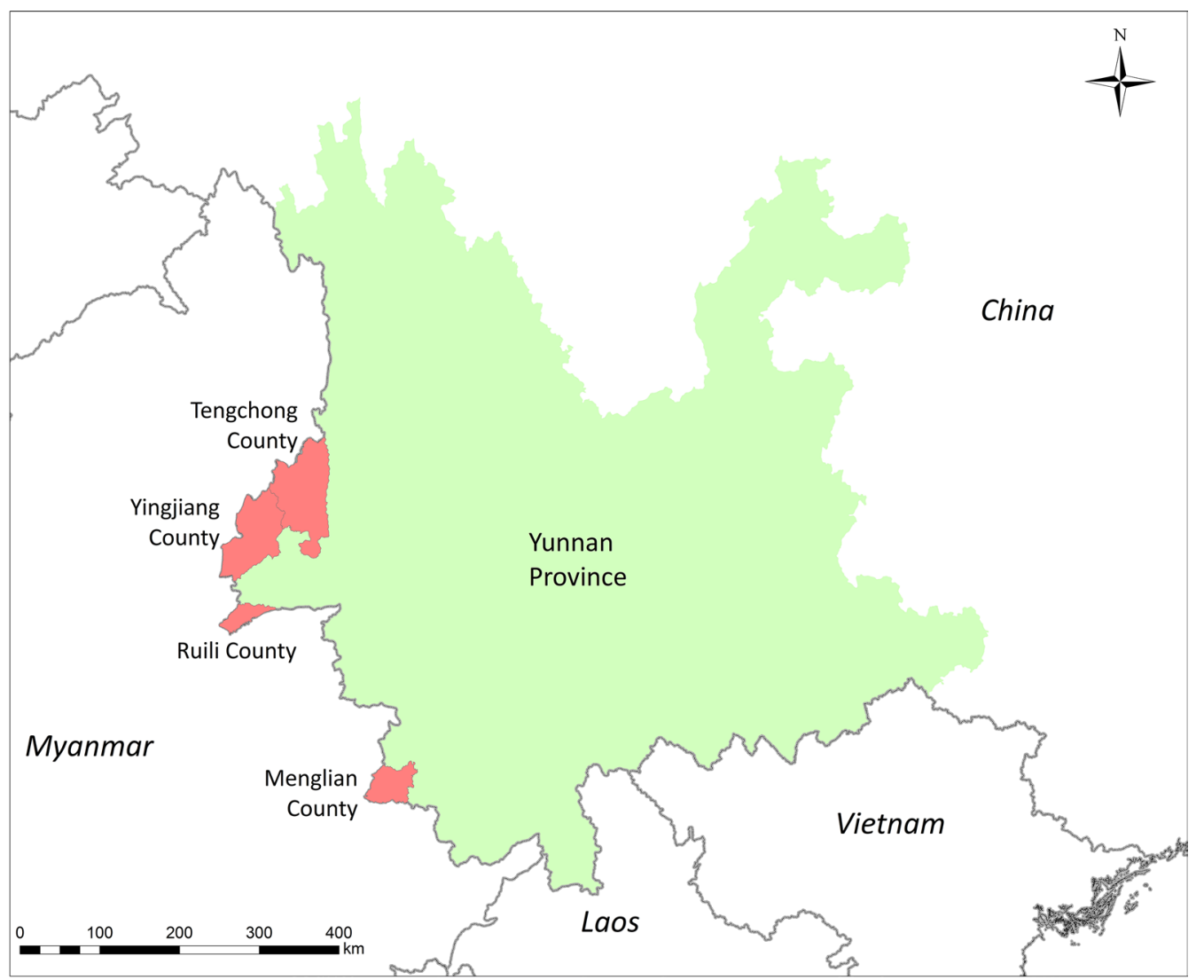

Fig. 1 Sentinel sites for therapeutic efficacy study in Yunnan Province from 2010 to 2014

measurement and thick/thin blood smear preparation and examination were performed at daily for the first 3 days with DHA-PPQ treatment to evaluate the day 3 positive rate. Post-discharge parasite examinations were performed on days 7, 14, 21, 28, 35 and 42. The parasite species and parasitaemia were identified by microscopists certified as Level 1 by the WHO. If the discrepancies of the two values were $>30 \%$, they were re-evaluated by a third microscopist to obtain a final diagnostic consensus (the average of the two close values of the parasitaemia).

\section{Clinical treatment outcome}

Treatment efficacy was evaluated based on clinical and parasitological outcomes and study end points in accordance with WHO guidelines for TES studies (Additional file 1). The outcomes were classified as early treatment failure (ETF), late clinical failure (LCF), late parasitological failure (LPF) and adequate clinical and parasitological response (ACPR). Primary endpoint was ACPR on day 42. PCR genotyping of $m s p 1, m s p 2$ and glurp, comparing day 0 and day of failure samples, was used to differentiate recrudescence (same parasite strain) from new infection with another strain parasite [26].

\section{$k 13$ sequencing and $p m 2$ amplification} Sample collection and DNA extraction

Dry blood spots on filter paper (Whatman ${ }^{\mathrm{TM}}$ 903, GE Healthcare, USA) were collected from participants from TES studies and clinical patients by passive surveillance from 2010 to 2014. All the filter paper samples were used for $k 13$ sequencing and $p m 2$ amplification. DNA was extracted from dried blood spots using QIAamp 96 DNA Blood Kit (Valencia, CA, USA).

\section{k13 sequencing}

A nested PCR amplification method (Takara PCR kit) to amplify the $k 13$ gene from codon 433 to 702 (850 bp) as described previously with some minor modifications [27]. The primers for nested PCR, sequencing PCR and cycling conditions for $k 13$ was shown in Additional file 2. PCR products were purified using filter plates (Edge Biosystems, Gaithersburg, MD, USA) and directly sequenced and analyzed on an ABI 3730XL automatic sequencer. The amplification products were analyzed by $1.5 \%$ agarose gel electrophoresis before sequencing. Purified products were sequenced by an ABI 3730XL automatic sequencer. Bi-directional sequencing was used and all 
the products were sequenced twice using independently amplified PCR products.

\section{pm2 amplification}

ASYBR-green based quantitative PCR (ThermoFisher Scientific, Waltham, MA, USA) was used to determine the copy number of pm2 (PF3D7_1408000) as previously described with modifications [24]. The copy numbers were quantified with quantitative PCR thermal cycle at $98{ }^{\circ} \mathrm{C}$ for $3 \mathrm{~min}$, followed by 45 cycles at $98{ }^{\circ} \mathrm{C}$ for $15 \mathrm{~s}$, $63{ }^{\circ} \mathrm{C}$ for $20 \mathrm{~s}$, and $72{ }^{\circ} \mathrm{C}$ for $20 \mathrm{~s}$ on a C1000 Thermal Cycler (Bio-Rad, Marnes-la-Coquette, France) with the CFX96 Real-Time System (Bio-Rad). All the procedures were executed in triplicate. Plasmodium falciparum $\beta$-tubulin (PF3D7_1008700) gene with single copy was used as the internal non-duplicated standard and the $P$. falciparum 3D7 clone as a parallel 1 copy control. The primers sequences for $p m 2$ and internal standard of $P$. falciparum $\beta$-tubulin were shown in Table 1 . The size of PCR products was $79 \mathrm{bp}$. The $P$. falciparum NF54 strain was also included in each amplification as the control. The copy number was calculated by the $2-\Delta \mathrm{Ct}$ method $\left(\triangle \mathrm{Ct}=\mathrm{Ct}_{P f P M 2}-\mathrm{Ct}_{P f \beta \text {-tubulin }}\right.$, where $\mathrm{Ct}$ is the threshold cycle). Copy number $>1.6$ was defined as amplification of the gene. The quantitative PCR primers are listed in Table 1 and protocols are shown in Additional file 3.

\section{Data analysis}

The outcome of the therapeutic efficacy study was evaluated with the WHO protocol [28]. The output sequence data of $k 13$ were assembled, edited, and aligned using Sequencher (version 5.1) software. PlasmoDB accession number PF3D7_1343700 (http://www.plasmodb.org) was used as a reference sequence. Fisher's exact test was used for statistical analysis. A regression test was used to estimate the association between the day 3 positive rate and $k 13$ mutation and the relationship of the pm2 copy number variants $(\mathrm{CNV})$ and $k 13$ mutations. Variables with $P<0.05$ were considered statistically significant.

\section{Ethical considerations}

This study was approved by the Institutional Review Board of the National Institute of Parasitic Diseases,
Chinese Center for Disease Control and Prevention and of the WHO Western Pacific Regional Office. Written informed consent was obtained from patients or guardians. The studies were also registered as clinical trials at https://www.anzctr.org.au under the numbers ACTRN12610001008011 and ACTRN12610001028099.

\section{Results}

\section{Therapeutic efficacy study}

A total of 174 participants received directly observed anti-malarial treatment with DHA-PPQ. The treatment outcome of DHA-PPQ was adjusted and corrected by PCR [29]. One hundred and forty of 174 participants completed the 42 days follow-up and 19.5\% (34/174) were withdrawn or lost to follow-up. The ACPR rates in Yingjiang of 2010 (20/20), 2012(43/43) and 2013(20/20), Tengchong in $2012(20 / 20)$ and Ruili in $2013(6 / 6)$ were all $100.0 \%$. The average ACPR rate of DHA-PPQ treatment in all the sites was $99.5 \%$ (minimum: 96.8\%; maximum: 100.0\%) (Table 2). One patient from Menglian County in 2014 showed treatment failure after DHA-PPQ treatment on day 35 was confirmed using PCR amplification as $P$. vivax infection. Another participant from Yingjiang County in 2012 experienced early treatment failure with parasitaemia and axillary temperature $\geq 37.5{ }^{\circ} \mathrm{C}$ on day 3 following treatment with DHA-PPQ, but this patient cleared parasitaemia without rescue treatment and was finally classified to be ACPR [26]. Seven of 174 participants (4.0\%) were positive for parasitaemia on day 3 after DHA-PPQ treatment.

\section{k13 sequencing}

The $k 13$ gene was successfully sequenced in 268 samples from therapeutic efficacy studies $(39.6 \%, 99 / 268)$ and the hospitals by passive surveillance $(63.1 \%, 169 / 268)$ (Table 3). Twelve non synonymous mutations (F446I, N458Y, Q467H, C469Y, F483S, F495L, P553L, E556D, $\mathrm{R} 561 \mathrm{H}, \mathrm{A} 578 \mathrm{~S}, \mathrm{~V} 589 \mathrm{G}$, and Q661R) in the $k 13$ propeller domain were observed in 118 samples, and the prevalence of $k 13$ mutations was $44.0 \%(118 / 268)$. The prevalence of $k 13$ mutation in TES samples and passive surveillance was 46.5\% (46/99) and 46.2\% (72/169), respectively. F446I was the predominant $k 13$ mutation

Table 1 Plasmodium falciparum pm2 copy number determination with listing of quantitative PCR primers and protocol

\begin{tabular}{|c|c|c|c|c|}
\hline Primer sequence & Sequences & $\operatorname{Tm}\left({ }^{\circ} \mathrm{C}\right)$ & Product size (bp) & $\begin{array}{l}\text { Range of melt } \\
\text { temperature } \\
\left({ }^{\circ} \mathrm{C}\right)\end{array}$ \\
\hline Pfpm2_CN_F & 5'-TGGTGATGCAGAAGTTGGAG-3' & 59.8 & 79 & $76.8-77.2$ \\
\hline Pfpm2_CN_R & 5'-TGGGACCCATAAATTAGCAGA-3' & 59.4 & & \\
\hline Pf $\beta$-tubulin_CN_F & 5'-TGATGTGCGCAAGTGATCC-3' & 61.9 & 79 & $79.0-79.2$ \\
\hline Pf $\beta$-tubulin_CN_R & 5'-TCCTTTGTGGACATTCTTCCTC-3' & 60.5 & & \\
\hline
\end{tabular}


Table 2 Outcome of the therapeutic efficacy study of DHA-PPQ treatment of uncomplicated P. falciparum from 2010 to 2014

\begin{tabular}{llllllll}
\hline Year & Sites & Day 3+(\%) & ACPR (\%) & LCF (\%) & LPF (\%) & ETF (\%) & WTH/LFU (\%) \\
\hline $2010^{c}$ & Yingjiang & $5.9 \%(1 / 29)$ & $100.0 \%(20 / 20)$ & $0.0 \%(0 / 20)$ & $0.0 \%(0 / 20)$ & $0.0 \%(0 / 20)$ & $31.0 \%(9 / 29)$ \\
$2012^{c}$ & Yingjiang & $3.9 \%(2 / 50)$ & $100.0 \%(43 / 43)^{\mathrm{a}}$ & $0.0 \%(0 / 43)$ & $0.0 \%(0 / 43)$ & $0.0 \%(0 / 43)$ & $14.0 \%(7 / 50)$ \\
$2012^{c}$ & Tengchong & $4.5 \%(1 / 22)$ & $100.0 \%(20 / 20)$ & $0.0 \%(0 / 20)$ & $0.0 \%(0 / 20)$ & $0.0 \%(0 / 20)$ & $9.1 \%(2 / 22)$ \\
2013 & Yingjiang & $9.5 \%(2 / 22)$ & $100.0 \%(20 / 20)$ & $0.0 \%(0 / 20)$ & $0.0 \%(0 / 20)$ & $0.0 \%(0 / 20)$ & $9.1 \%(2 / 22)$ \\
2013 & Ruili & $0.0 \%(0 / 11)$ & $100.0 \%(6 / 6)$ & $0.0 \%(0 / 6)$ & $0.0 \%(0 / 6)$ & $0.0 \%(0 / 6)$ & $45.5 \%(5 / 11)$ \\
2014 & Menglian & $2.5 \%(1 / 40)$ & $96.8 \%(30 / 31)$ & $3.1 \%(1 / 31)^{b}$ & $0.0 \%(0 / 31)$ & $0.0 \%(0 / 31)$ & $22.5 \%(9 / 40)$ \\
\hline
\end{tabular}

ACPR, adequate clinical parasitological response; LCF, late clinical failure; LPF, late parasitological failure; ETF, early treatment failure; WTH, withdrawal; LFU, loss to follow-up

a One of 43 participants had parasite and fever on day 3, but this case was continually followed up and cleared parasitaemia without rescue treatment and was finally classified to be ACPR

b P. vivax infection was positive on day 35

c Part data of the therapeutic efficacy study of DHA-PPQ has been presented in another study [7]

and accounted for $74.6 \%(88 / 118)$ of all $k 13$ mutations. The $k 13$ mutation from samples collected from Yingjiang and Tengchong had a higher prevalent than from Ruili and Menglian. In addition, five of the seven patients who were positive for parasitaemia on day 3 after DHA-PPQ treatment were observed with $k 13$ mutation $(\mathrm{N} 458 \mathrm{Y}$, C469Y, and P553L). The k13 mutation was not significantly associated with the day 3 positive parasitaemia after DHA-PPQ treatment $(P=0.245)$.

\section{pm2 copy number variation}

The 224 samples comprising 91 with $k 13$ mutant and 133 with $k 13$ wild-type were tested on $p m 2$ copy number amplification (Table 3). Proportion of different $k 13$ genotype of the samples for $p m 2$ amplification was shown in Fig. 2. Only one sample was observed with multi-copies of pm2 from $k 13$ mutant samples and other $k 13$ mutant or wild-type samples showed no copy variants of pm2 $(99.6 \%, 223 / 224)$. These data were consistent with the therapeutic efficacy of DHA-PPQ. Since there was only one sample with $p m 2$ multiple copies, the data did not meet the conditions for statistical analysis of the association between the pm 2 copy number variants and $k 13$ mutations. Nevertheless, it was necessary to note that this sample with multi-copies of $p m 2$ was also carrying k13 mutation.

\section{Discussion}

The emergence and rapid spread of multidrug-resistant $P$. falciparum across the GMS is a threat to the therapeutic efficacy of DHA-PPQ for treatment of uncomplicated $P$. falciparum. According to the WHO definition, the partial resistance to artemisinin is characterized as much slower clearance of parasitaemia in the first 3 days of treatment following artemisinin mono-therapy or ACT [30]. Such resistance does not usually lead to treatment failure; however, if the artemisinin component is less effective, the partner drug has to clear a greater parasitic mass, jeopardizing the future efficacy of the partner drug. PPQ resistance was first reported in Cambodia [20]. Recently, alarmingly high rates of treatment failure have occurred in Cambodia, Vietnam, and Thailand, which will have contributed to increased transmission of $P$. falciparum [21].

In Africa, artemisinin resistance emergence has been reported [31], but artemisinin (partial) resistance has not been confirmed to date and first-line ACT remains efficacious in all malaria-endemic settings [32-34]. DHA-PPQ was increasingly deployed as an anti-malarial drugs in Africa. The $p m 2$ duplications was observed in recurrent infections in Mali within 2 months after DHA-PPQ treatment [35]. Another study reported that only a single copy of $p m 2$ was detected in two isolates from Ethiopia and Cameroon after DHA-PPQ failures [36, 37]. These findings indicated the DHA-PPQ failures or pm2 multi-copies in very few cases in Africa, but still raised concerns about the long-term efficacy of DHA-PPQ treatment.

In a previous study, $P$. falciparum showed markedly delayed clearance following 7 days' treatment with artesunate along the China-Myanmar border, and k13 F446I was the predominant mutation and was associated with delayed parasite clearance [7]. The present study aimed to evaluate the therapeutic efficacy of DHA-PPQ, which is the most widely used artemisininbased combinations in China, as the first-line drug for uncomplicated P. falciparum infection. DHA-PPQ was still efficacy in Southern Yunnan Province with $>95 \%$ ACPR outcome. In the 1970s to 1980 s, PPQ alone was used for prophylaxis and treatment in the malaria endemic areas in Southern China [15, 38]. In 2009, PPQ was used as a partner drug of artemisinin for first-line treatment of $P$. falciparum infection in China and now 


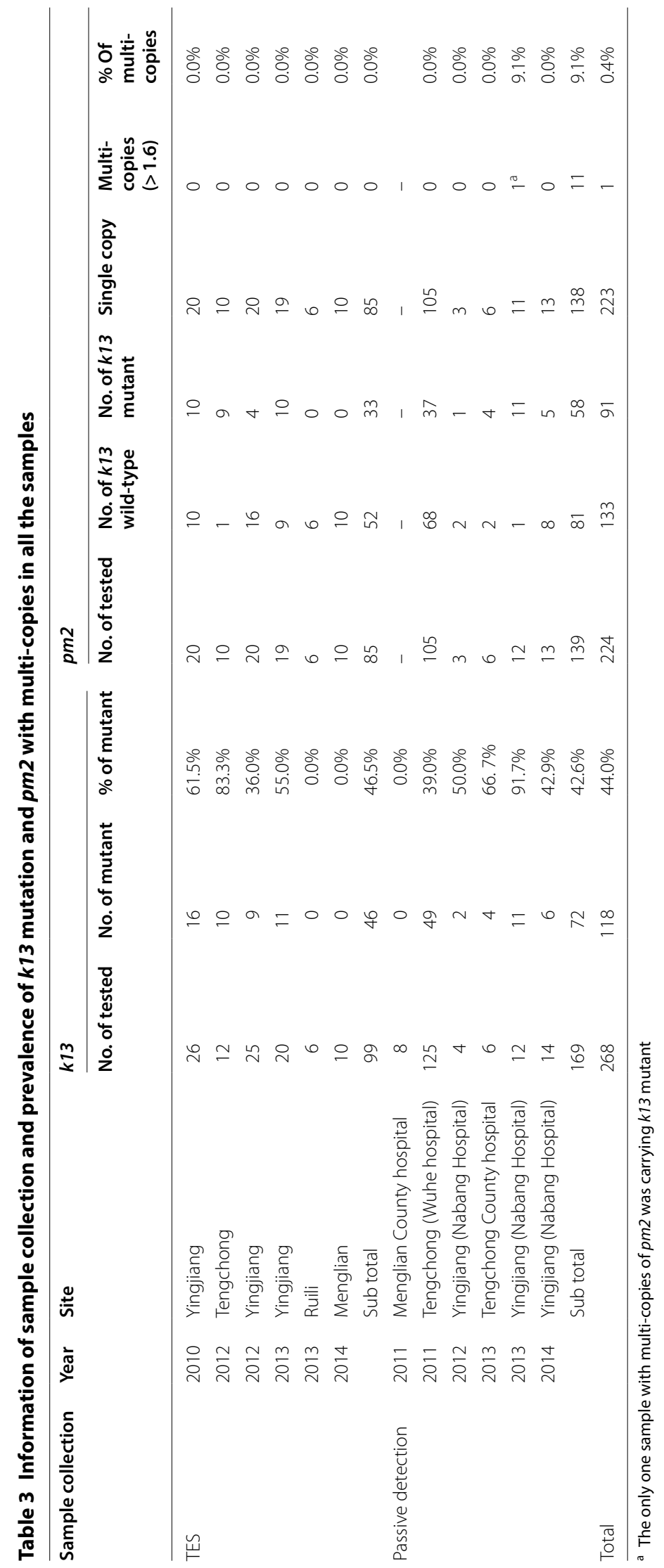




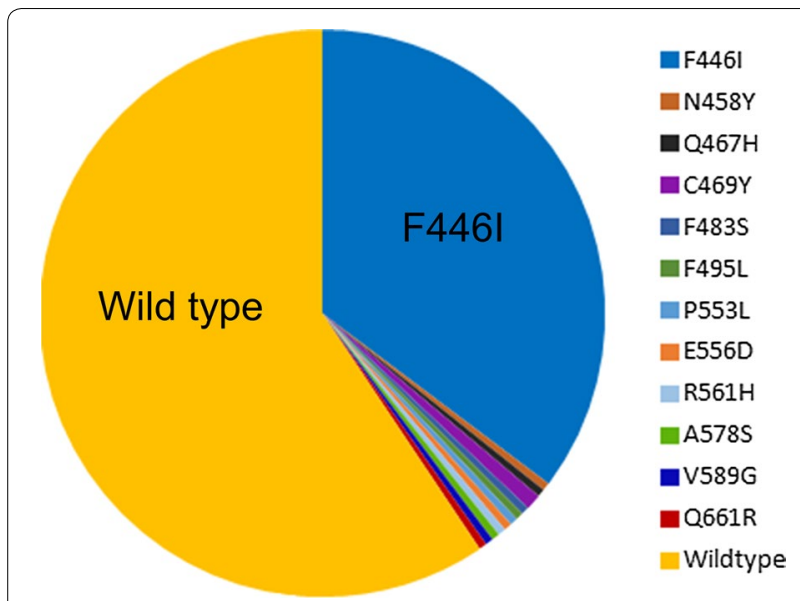

Fig. 2 Proportion of $k 13$ polymorphisms of the samples tested on pm2 amplification

DHA-PPQ is the most widely used artemisinin-based combination. Although no resistance to artemisinin and its partner drug PPQ was confirmed in falciparum malaria in Yunnan Province, the efficacy of ACT was decreasing. The prevalence of day 3 positive parasitaemia was $>3 \%$ in most of the sentinel sites and artemisinin resistance has been confirmed along the China-Myanmar border [7, 13].

Only one sample was observed with $p m 2$ multi-copies with $k 13$ mutation out of 91 samples and no copy variants of $k 13$ wild-type samples. The association between $k 13$ polymorphisms and $p m 2$ amplification was strong in the Cambodian parasites, which reflected the history of drug selection in Cambodia [24]. The isolates from the China-Myanmar border in our study showed high prevalence of single copy of $p m 2$ compared with the high multi-copies in Cambodia, which also indicated the low connectivity of genetic population between parasites from the China-Myanmar border and Cambodia [39]. Furthermore, the data for $p m 2$ copy number variants provide evidence that no PPQ resistance has emerged along the China-Myanmar border, which is consistent with the therapeutic efficacy of DHA-PPQ.

China is approaching malaria elimination and zero indigenous cases were reported from 2017 [25, 40]. There are still several challenges in the post malaria elimination stage. One big challenge is how to maintain surveillance and response capacity. Monitoring the efficacy of antimalarial drugs for the treatment of imported cases was recommended by the WHO in "A framework for malaria elimination" [41], which was called integrating drug efficacy surveillance (iDES). China has set up an antimalarial drug surveillance network that is responsible for implementing iDES of DHA-PPQ and anti-malarial molecular markers surveillance throughout the country.

\section{Limitations}

This study only analyzed the $p m 2$ copy number variants of $P$. falciparum isolates along the China-Myanmar border. Recent studies have identified that mutations in $P$. falciparum chloroquine resistance transporter (pfcrt) could contribute to a multi-factorial basis of PPQ resistance [42-44], which indicates that there are multi-genes or multi-mutations involved in PPQ resistance. Therefore, further molecular surveillance of PPQ resistance including multi-genes will be necessary.

\section{Conclusion}

DHA-PPQ for uncomplicated $P$. falciparum infection was still efficacy in the area with artemisinin-resistant malaria along the China-Myanmar border. There was no evidence to show PPQ resistance in the clinical study and molecular markers survey of $p m 2$. Continued monitoring of the parasite population using molecular markers will be important to track emergence and spread of resistance in this geographic region.

\section{Supplementary information}

Supplementary information accompanies this paper at https://doi. org/10.1186/s12936-020-03410-6.

Additional file 1. Classification of treatment outcomes in the therapeutic study.

Additional file 2: Table S1. Primers for nested PCR and sequencing PCR and cycling conditions for $k 13$.

Additional file 3. The protocol for pm2 copy number amplification.

\section{Acknowledgements}

We thank all the participants involved in the clinical study for their contribution work for the study, and local staff from Yingjiang Center for Disease Control and Prevention (CDC), Tengchong CDC, Ruili CDC and Menglian CDC.

\section{Meetings}

This study was presented at the annual meeting of the American Society of Tropical Medicine and Hygiene in Baltimore, MD, November 2017.

\section{Authors' contributions}

FH, ST and CVP conceived and designed the study; HL, LHT, SZ carried out the TES study; $\mathrm{HL}$ collected the passive detection samples; $\mathrm{FH}, \mathrm{BS}, \mathrm{XZ}$ carried out the data analysis; $\mathrm{BS}$ and FH conducted the laboratory work. PR, MMN, and CVP provided technique support for the data analysis. FH drafted the manuscript. All authors read and approved the final manuscript.

\section{Funding}

This work was supported by the Natural Science Foundation of Shanghai (No. 18ZR1443400), the National Important Scientific \& Technological Project 2018ZX10101002-002), the WHO Mekong Malaria Programme (WP/08/ MVP/000512 and WP/10/MVP/005837), NIH R01-Al125579 and the Overseas Public Health Training Programme of the Shanghai Health Bureau (Grant GWHW201210). 


\section{Competing interests}

The authors declare no competing interests. PR is a staff member of the World Health Organization. The author alone is responsible for the views expressed in this publication and does not necessarily represent the decisions, policy or views of the World Health Organization.

\section{Author details}

${ }^{1}$ National Institute of Parasitic Diseases, Chinese Center for Disease Control and Prevention, Shanghai, People's Republic of China. ${ }^{2}$ Yunnan Institute of Parasitic Diseases, Puer, People's Republic of China. ${ }^{3}$ Center for Vaccine Development and Global Health, University of Maryland School of Medicine, Baltimore, MD, USA. ${ }^{4}$ Global Malaria Programme, World Health Organization, Geneva, Switzerland. ${ }^{5}$ Duke Global Health Institute, Duke University, Durham, NC, USA.

Received: 23 June 2020 Accepted: 4 September 2020 Published online: 14 September 2020

\section{References}

1. WHO. World malaria report 2019. Geneva: World Health Organization; 2019.

2. Nosten F, White NJ. Artemisinin-based combination treatment of falciparum malaria. Am J Trop Med Hyg. 2007;77:181-92.

3. WHO. Guidelines for the treatment of malaria. 3rd ed. Geneva: World Health Organization; 2015.

4. Menard D, Khim N, Beghain J, Adegnika AA, Shafiul-Alam M, Amodu O, et al. A worldwide map of Plasmodium falciparum K13-propeller polymorphisms. N Engl J Med. 2016;374:2453-64.

5. Takala-Harrison S, Jacob CG, Arze C, Cummings MP, Silva JC, Dondorp $\mathrm{AM}$, et al. Independent emergence of artemisinin resistance mutations among Plasmodium falciparum in Southeast Asia. J Infect Dis. 2015;211:670-9.

6. Ashley EA, Dhorda M, Fairhurst RM, Amaratunga C, Lim P, Suon S, et al. Spread of artemisinin resistance in Plasmodium falciparum malaria. N Engl J Med. 2014;371:411-23.

7. Huang F, Takala-Harrison S, Jacob CG, Liu H, Sun X, Yang H, et al. A single mutation in $\mathrm{K} 13$ predominates in Southern China and is associated with delayed clearance of Plasmodium falciparum following artemisinin treatment. J Infect Dis. 2015;212:1629-35.

8. WHO. Strategy for malaria elimination in the GMS (2015-2030). Geneva: World Health Organization; 2015.

9. WHO. Status report: artemisinin resistance and artemisinin-based combination therapy efficacy. Geneva: World Health Organization; 2018.

10. WHO. Guidelines for the treatment of malaria. 2nd ed. Geneva: World Health Organization; 2010.

11. NHFPC. Technical regulation for application antimalarials of China. Beijing: National Health and Family Planning Commission of China; 2016.

12. Fairhurst RM. High antimalarial efficacy of dihydroartemisinin-piperaquine on the China-Myanmar border: the calm before the storm. Am J Trop Med Hyg. 2015;93:436-7.

13. Wang Y, Yang Z, Yuan L, Zhou G, Parker D, Lee MC, et al. Clinical efficacy of dihydroartemisinin-piperaquine for the treatment of uncomplicated Plasmodium falciparum malaria at the China-Myanmar border. Am J Trop Med Hyg. 2015;93:577-83

14. Liu H, Yang HL, Tang LH, Li XL, Huang F, Wang JZ, et al. In vivo monitoring of dihydroartemisinin-piperaquine sensitivity in Plasmodium falciparum along the China-Myanmar border of Yunnan Province, China from 2007 to 2013. Malar J. 2015;14:47.

15. Wang SQ, Christophel E, Lin SG, Meng F, Hu XM, Wang GZ, et al. Efficacy of dihydroartemisinin-piperaquine and artemether-lumefantrine in the treatment of uncomplicated falciparum malaria in Hainan, China. Zhongguo Ji Sheng Chong Xue Yu Ji Sheng Chong Bing Za Zhi. 2008;26:50-2 (in Chinese)

16. Phuc BQ, Rasmussen C, Duong TT, Dong LT, Loi MA, Menard D, et al. Treatment failure of dihydroartemisinin/piperaquine for Plasmodium falciparum malaria, Vietnam. Emerg Infect Dis. 2017;23:715-7.

17. Chaorattanakawee S, Lon C, Jongsakul K, Gawee J, Sok S, Sundrakes S, et al. Ex vivo piperaquine resistance developed rapidly in Plasmodium falciparum isolates in northern Cambodia compared to Thailand. Malar J. 2016;15:519.

18. Leang R, Taylor WR, Bouth DM, Song L, Tarning J, Char MC, et al. Evidence of Plasmodium falciparum malaria multidrug resistance to artemisinin and piperaquine in Western Cambodia: dihydroartemisinin-piperaquine open-label multicenter clinical assessment. Antimicrob Agents Chemother. 2015:59:4719-26.

19. Amato $R$, Lim $P$, Miotto $O$, Amaratunga $C$, Dek $D$, Pearson $R D$, et al. Genetic markers associated with dihydroartemisinin-piperaquine failure in Plasmodium falciparum malaria in Cambodia: a genotype-phenotype association study. Lancet Infect Dis. 2017;17:164-73.

20. Saunders DL, Vanachayangkul P, Lon C, Program USAMMR, National Center for Parasitology E, Malaria C, et al. Dihydroartemisinin-piperaquine failure in Cambodia. N Engl J Med. 2014;371:484-5.

21. Van der Pluijm RW, Imwong M, Chau NH, Hoa NT, Thuy-Nhien NT, Thanh $\mathrm{NV}$, et al. Determinants of dihydroartemisinin-piperaquine treatment failure in Plasmodium falciparum malaria in Cambodia, Thailand, and Vietnam: a prospective clinical, pharmacological, and genetic study. Lancet Infect Dis. 2019;19:952-61.

22. Hamilton WL, Amato R, van der Pluijm RW, Jacob CG, Quang HH, ThuyNhien NT, et al. Evolution and expansion of multidrug-resistant malaria in southeast Asia: a genomic epidemiology study. Lancet Infect Dis. 2019;19:943-51.

23. Rovira-Vallbona E, Van Hong N, Kattenberg JH, Huan RM, Hien NTT, Ngoc $\mathrm{NTH}$, et al. Efficacy of dihydroartemisinin/piperaquine and artesunate monotherapy for the treatment of uncomplicated Plasmodium falciparum malaria in Central Vietnam. J Antimicrob Chemother. 2020;75:2272-81.

24. Witkowski B, Duru V, Khim N, Ross LS, Saintpierre B, Beghain J, et al. A surrogate marker of piperaquine-resistant Plasmodium falciparum malaria: a phenotype-genotype association study. Lancet Infect Dis. 2017:17:174-83.

25. Feng J, Zhang L, Huang F, Yin JH, Tu H, Xia ZG, et al. Ready for malaria elimination: zero indigenous case reported in the People's Republic of China. Malar J. 2018;17:315.

26. WHO. Methods and techniques for clinical trials on antimalarial efficacy: genotyping to identify parasite populations. Geneva: World Health Organization; 2008

27. Mbengue A, Bhattacharjee S, Pandharkar T, Liu H, Estiu G, Stahelin RV et al. A molecular mechanism of artemisinin resistance in Plasmodium falciparum malaria. Nature. 2015;520:683-7.

28. WHO. Methods for the surveillance of antimalarial drug efficacy. Geneva: World Health Organization; 2009.

29. Snounou G, Viriyakosol S, Zhu XP, Jarra W, Pinheiro L, do Rosario VE, et al. High sensitivity of detection of human malaria parasites by the use of nested polymerase chain reaction. Mol Biochem Parasitol. 1993;61:315-20.

30. Menard D, Dondorp A. Antimalarial drug resistance: a threat to malaria elimination. Cold Spring Harb Perspect Med. 2017;7:a025619.

31. Lu F, Culleton R, Zhang M, Ramaprasad A, von Seidlein L, Zhou H, et al. Emergence of indigenous artemisinin-resistant Plasmodium falciparum in Africa. N Engl J Med. 2017;376:991-3.

32. Taylor SM, Parobek CM, DeConti DK, Kayentao K, Coulibaly SO, Greenwood BM, et al. Absence of putative artemisinin resistance mutations among Plasmodium falciparum in Sub-Saharan Africa: a molecular epidemiologic study. J Infect Dis. 2015;211:680-8.

33. Roper C, Alifrangis M, Ariey F, Talisuna A, Menard D, Mercereau-Puijalon $\mathrm{O}$, et al. Molecular surveillance for artemisinin resistance in Africa. Lancet Infect Dis. 2014;14:668-70.

34. Bastiaens GJ, Bousema T, Leslie T. Scale-up of malaria rapid diagnostic tests and artemisinin-based combination therapy: challenges and perspectives in sub-Saharan Africa. PLoS Med. 2014;11:e1001590.

35. Inoue J, Silva M, Fofana B, Sanogo K, Martensson A, Sagara I, et al. Plasmodium falciparum plasmepsin 2 duplications, West Africa. Emerg Infect Dis. 2018;24:1591-3.

36. Malvy D, Torrentino-Madamet M, L'Ollivier C, Receveur MC, Jeddi F,

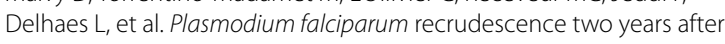
treatment of an uncomplicated infection without return to an area where malaria is endemic. Antimicrob Agents Chemother. 2018;62:e01892-17.

37. Russo G, L'Episcopia M, Menegon M, Souza SS, Dongho BGD, Vullo V, et al. Dihydroartemisinin-piperaquine treatment failure in uncomplicated Plasmodium falciparum malaria case imported from Ethiopia. Infection. 2018:46:867-70. 
38. Zhang CL, Zhou HN, Wang J, Liu H. In vitro sensitivity of Plasmodium falciparum isolates from China-Myanmar border region to chloroquine, piperaquine and pyronaridine. Zhongguo Ji Sheng Chong Xue Yu Ji Sheng Chong Bing Za Zhi. 2012;30:41-4 (in Chinese).

39. Shetty AC, Jacob CG, Huang F, Li Y, Agrawal S, Saunders DL, et al. Genomic structure and diversity of Plasmodium falciparum in Southeast Asia reveal recent parasite migration patterns. Nat Commun. 2019;10:2665.

40. Feng $X$, Levens J, Zhou XN. Protecting the gains of malaria elimination in China. Infect Dis Poverty. 2020:9:43.

41. WHO. A framework for malaria elimination. Geneva: World Health Organization; 2017.

42. Silva M, Calcada C, Teixeira M, Veiga MI, Ferreira PE. Multigenic architecture of piperaquine resistance trait in Plasmodium falciparum. Lancet Infect Dis. 2020;20:26-7.
43. Agrawal S, Moser KA, Morton L, Cummings MP, Parihar A, Dwivedi A, et al. Association of a novel mutation in the Plasmodium falciparum chloroquine resistance transporter with decreased piperaquine sensitivity. J Infect Dis. 2017;216:468-76.

44. Dhingra SK, Redhi D, Combrinck JM, Yeo T, Okombo J, Henrich PP, et al. A variant PFCRT isoform can contribute to Plasmodium falciparum resistance to the first-line partner drug piperaquine. mBio. 2017;8:e00303-17.

\section{Publisher's Note}

Springer Nature remains neutral with regard to jurisdictional claims in published maps and institutional affiliations.
Ready to submit your research? Choose BMC and benefit from:

- fast, convenient online submission

- thorough peer review by experienced researchers in your field

- rapid publication on acceptance

- support for research data, including large and complex data types

- gold Open Access which fosters wider collaboration and increased citations

- maximum visibility for your research: over 100M website views per year

At BMC, research is always in progress.

Learn more biomedcentral.com/submissions 